\title{
Considerazioni attorno ad una questione metricologica. Il Boccaccio e le origini dell'ottava rima
}

\author{
Lorenzo Bartoli \\ Universidad Autónoma de M adrid
}

\begin{abstract}
In this article the Author offers a critical review of the mejor works of investigation on the issue of the creation and establishing of a specific poetic metre, the ottava rima and on the role played by Giovanni Boccaccio in such an issue. The Author argues that besides all the efforts to establish an origin for such a metre afore Boccaccio's poems, the results have been inconclusive; whereas Boccaccio's preminance in the establishing of the use of such a metre in Tuscany seems to grow in evidence slowly bur surely so that he should still be considered, de facto, if not the inventor, at least the designer of the original ottava.
\end{abstract}

Le questioni metricologiche, soprattutto quelle che riguardano l'origine di un particolare metro, nascondono in filigrana (almeno quando sono ben condotte) l'eco di ben più ampi dibattiti. D ietro all'invenzione della terza rima, ad esempio, non ritroviamo semplicemente dispiegate le doti filologiche che ci consentono di formulare un nome - quello di D ante Alighieri - ad indicare il pater storico di tale metro; bensì si ricompongono, attorno a $\mathrm{D}$ ante ed alla sua figura, tutti i tasselli che appartengono alle stagioni originarie di una letteratura. Sicché la paternità dantesca non sarà suffragata solo da riflessioni orientate in senso storico-filologico (la Commedia di D ante è la prima opera nella storia della letteratura italiana in cui viene usata la terza rima), ma si allargherà a comprendere altri elementi legati a $\mathrm{D}$ ante ed alla sua fortuna, quali le sue peculiari doti di innovazione sul terreno del genere letterario (Vita N uova e D e vulgari eloquentia, innanzitutto), insieme ad uno spiccato sperimentalismo nel terreno più precipuamente poetico. M a soprattutto, si direbbe, la paternità dantesca viene suffragata dalla tradizione che accompagnò la terza rima dopo l'esperienza dantesca e che in pratica appare tutta condizionata dal magistero del'Alighieri. In altre parole, le argomentazioni a favore della paternità di D ante per ciò che riguarda le origini della terza rima si compongono di due elementi separati: da una parte la documentazione filologica concernente la prima attestazione di tale metro; dall'altra lo sviluppo del metro stesso, la sua tradizione. 
O ra, per quanto riguarda le origini dell'ottava rima, valgono le premesse fin qui fatte. Non vi è dubbio, infatti, che insieme al dibattito sulle origini di tale metro ed attorno alla presunta paternità del Boccaccio è nata e si è sviluppata una ben più ampia discussione intorno alle origini della tradizione canterina in ottave. Benché, infatti, gli el ementi filologici a nostra disposizione per dirimere la questione siano pressoché gli stessi da circa cent'anni a questa parte (nella sostanza: è chiaro, poi, che precisazioni e nuove messe a punto, soprattutto per i capitoli della storia che riguardano il Boccaccio, sono invece abbastanza numerose e significative); e benché la pratica canterina pre-boccaccesca toscana continui ad essere poco più che un'ipotesi di lavoro (particolarmente per quel che riguarda la struttura metrica), le interpretazioni continuano ad essere molteplici. Cosi, sullo sfondo di ciascuna argomentazione soggiacciono implicazioni critiche e metodologiche che vanno ben aldilà degli studi sulla letteratura canterina. Le osservazioni del Dionisotti, del Roncaglia, del Gorni, del Balduino (per citare i lavori, a nostro giudizio, più significativi degli ultimi quarant'anni di studi), ${ }^{1}$ pur non riuscendo a dirimere la vexata quaestio circa l'origine dell'ottava rima, hanno tuttavia arricchito la nostra percezione dell'ideologia letteraria tre-quattrocentesca, definendola insieme nella sua ampiezza geografica e nel suo sviluppo storico e stilistico.

Sicché, converrà tornare a fare il punto su tale questione, segnalando quelle che appaiono essere state le linee maestre della critica letteraria e della filologia dell'ultimo secolo, da quando, in definitiva, il Rajna e con lui altri rappresentanti della scuola storica (il Flamini, in particolare) dichiararono, senza mezzi termini, che l'uso dell'ottava rima toscana nel Boccaccio derivava dall'imitazione della letteratura canterina e non viceversa. Scriveva infatti il Rajna: ${ }^{2}$

La tradizione degli eruditi, che la voleva invenzione del Boccaccio [sintendel'ottava rima] e adoperata per la prima volta nella Tesei de, I'anno di grazia 1341, va messa senz'altro fra le anticaglie.

1. I riferimenti sono a Carlo D ION ISOTTI, «Entrée d'Espagne, Spagna, Rotta di Roncisvalle», Studi in onore di A. M onteverdi, M odena: ST EM , 1959, p. 207-241; ID., «Appunti su testi antichi», Italia medievale e umanistica, n. 7, 1964, p. 77-131; Aurelio Ron CAGLIA, «Per la storia dell'ottava rima», Cultura neolatina, n. 25, 1965, p. 5-14; G uglielmo G o RN I, «Un'ipotesi sulle origini dell'ottava rima», M etrica, n. 1, 1978, p. 80-94; Armando BALD UIN 0 , (a cura di), Cantari del Trecento, M ilano: M arzorati, 1970; I ., Boccaccio, Petrarca e altri poeti del Trecento, Firenze: O Ischki, 1984. Andranno inoltre ricordati gli studi di Limentani (Alberto LIM ENTAN I, ¿Struttura e storia dell'ottava rima», Lettere italiane, n. 13, 1961, p. 20-77) e di Picone (M ichelangelo PICONE, «Boccaccio ela codificazione dell'ottava», in M. Cottin o-Jones e E.F. T ut tle (a cura di), Boccaccio: Secoli di vita, Ravenna: Longo, 1977, p. 53-65), i quali, tuttavia, non rientrano immediatamente nell'ambito del nostro discorso critico.

2. Cfr. Pio RajnA, Lefonti del'O rlando furioso, ristampa della 2. ed. 1990 accresciuta d'inediti, a cura di F. M Azzo N I, Firenze: Sansoni, 1975, p. 16 (la prima edizione del fondamentale studio del Rajna risale al 1876). 
$E$, precisava il Flamini, «D a un pezzo è dimostrata erronea ed assurda l'opinione che dell'ottava rima faceva inventore il Boccaccio» ${ }^{3}$

D i fatto, dietro al ragionamento che muoveva il Boccaccio dalla sede di inventore dell'ottava non intervenivano tanto osservazioni di carattere storico-paleografico, quanto piuttosto le indicazioni di una filologia che, nello forzo della ricostruzione della tradizione cavalleresca ed in particolare di quella ariostesca, finiva per spostare arbitrariamente indietro le origini stesse di tale tra dizione canterina in ottava. O Itre le indicazioni che venivano fornite dai manoscritti, la genesi dei cantari in ottave veniva retrodatata secondo un'analisi tutta interna ai testi stessi (si veda ad esempio il caso della Ponzela Gaia): una pratica, questa, che naturalmente si rifletteva in un ripensamento della questione metricologica, nel senso che la retrodatazione ai primi del Trecento di alcuni cantari in ottava rima, mentre escludeva inevitabilmente l'ipotesi di conside rare il Boccaccio quale inventore di questo metro, finiva per ricondurre la genesi dell'ottava in un ambito decisamente popolare.

Cosi, gli studi che condusse il D ionisotti negli anni Cinquanta e Sessanta - che puntavano innanzitutto ad una ridiscussione metodologica attorno alle strategie filologiche ereditate dalla scuola storica, particolarmente con riferimento alla questione della datazione dei primi cantari in ottave - finirono per investire il punto nevral gico del dibattito, ovvero la posizione del Boccaccio in merito all'invenzione dell'ottava rima. In sostanza il D ionisotti, nel ripristinare per le datazioni dei cantari i valori della paleografia (secondo un'argomentazione filologica che venne poi precisata, nella metodologia ecdotica, dal D e Robertis), ${ }^{4}$ spostava in basso e cioè decisamente verso la seconda metà del Trecento e poi, per i cantari lunghi, verso il Q uattrocento, le prime testimonianza dirette di cantari in ottava: da cui, evidentemente, scaturiva un decisivo recupero della centralità del Boccaccio nel decorso di tale tradizione, giacché al C ertaldese si doveva la prima attestazione di una ottava toscana narrativa sicuramente formata, quella del Filostrato, 1335 (ma sulla datazione delle opere del Boccaccio, e particolarmente su quella del Filostrato stesso, anche in rapporto al Filocolo, torneremo più avanti). In definitiva, pareva che anche per le origini dell'ottava rima ci si potesse affidare ad un nome di sicuro prestigio, che garantisse, per cosi dire, un principio legato alla cultura alta, se non altissima, per le successive imprese poetiche del Pulci, del Boiardo e dell'Ariosto. In definitiva, Boccaccio come una sorta di alter D ante.

Le osservazioni che a suo tempo apportò il Roncaglia («Per la storia delI'ottava rima»), circa una genesi francese dell'invenzione del Boccaccio, al quale significativamente si deve non solo la prima attestazione di ottava di tipo toscano ( $A B A B A B C C$ ), ma anche la prima ottava di tipo siciliano, ovvero

3. C fr. Francesco Flam In I, Studi di storia letteraria italiana e straniera, Livorno: Giusti, 1895, p. 151.

4. II riferimento è, naturalmente, a D omenico D E ROBERTIS, «Problemi di metodo nell'edizione dei cantari», Studi e problemi di critica testuale, Bologna: Commissione per i testi di lingua, 1961, p. 119-138. 
$A B A B A B A B^{5}$ (e precisamente il Roncaglia pensava ad una linea che viaggiava attraverso la N apoli angioina); nonché la ricostruzione proposta dal G orni, incentrata sul Boccaccio come elaboratore di un metro di canzone ciniana, rafforzano e precisano l'ipotesi del D ionisotti (ed in qualche modo ne cercano di completare - se non di correggere su certi aspetti - la pars construens) circa una genesi evidentemente colta dell'ottava rima e dell'area poetica dalla quale l'avrebbe ricavata il Boccaccio; e tuttavia entrambe le ipotesi, del Roncaglia e del Gorni, stentano a riguadagnare il respiro e lo sguardo a tutto campo che aveva l'indagine del Dionisotti e restano, per cosi dire, sacrificate in un ambito troppo da laboratorio letterario (ché se non è probabile, come osservava lo stesso Gorni, che un metro rimanga inattivo per un buon settantennio come implicherebbe l'ipotesi del Roncaglia - ; nemmeno è probabile che un metro destinato alla narrazione ed alla serie ripetuta di endecasillabi derivi, per alchimia poetica, dalla struttura mista di endecasillabi e settenari della canzone ciniana, secondo le indicazioni del proprio G orni).

II fatto centrale, tuttavia, è che l'idea del D ionisotti, mentre vale metodologicamente, lamenta viceversa un'eccessiva concentrazione attorno alla figura del Boccaccio. U na volta accertato filologicamente che andava fatta risalire al Boccaccio stesso la prima attestazione di tale metro (e che anzi al C ertal dese rimontava anche la prima attestazione dell'ottava di tipo siciliano, come si diceva), la storia o la preistoria, per meglio dire, dell'ottava è stata letta tutta in funzione del Boccaccio, e dunque al di fuori della tradizione canterina vera e propria. $\mathrm{D}$ a ciò derivò un pregiudizio critico, che per quanto sfumato e non sempre ben individuabile, ha fatto si che tutta la tradizione canterina - intendendo qui sia la fase preboccaccesca che quella postboccaccesca - assumesse una connotazione piuttosto statica e tutto sommato distorta rispetto alla fluidità dei dati che sono giunti fino a noi. In altre parole, il Boccaccio senz'altro contribuì in modo decisivo allo sviluppo del genere: ma tale contributo, specialmente in senso metricologico, sarà apprezzabile filologicamente solo a condizione di inserirlo appunto nelle giuste coordinate del genere. II che significa che l'attenzione deve essere rivolta non tanto alla stagione dei poemi boccacceschi in sé; quanto piuttosto, allo scarto che quella stagione rappresentò nella traiettoria complessiva della tradizione canterina.

Da questo punto di vista, cosi pare, la ricostruzione del D ionisotti risulta poco convincente per una ragione molto precisa, ché volendo fare del Boccaccio e dell'ottava una ragione critica analoga, se non identica a quella di $D$ ante e della terza rima, non tiene conto dell'attività del Boccaccio nella prospettiva della tradizione canterina; manca cioè, I'analisi della traiettoria metrica non

5. Ci si riferisce all'epitaffio di Giulia Topazia romana, contenuto nel Filocolo, segnalato per primo dal Wilkins nel 1956 (cfr. E.H. W ILKIN S, «Boccaccio's First O ctave», I talica, n. 33, 1956, p. 19) e per il quale il D ionisotti (Appunti, cit.) pensa, coerentemente con la sua interpretazione, ad una tradizione al ta e precisamente all'imitazione dei distici elegiaci degli epigrammi latini. 
dell'ottava boccaccesca, ma dell'ottava canterina intesa come testimonianza di una tradizione che certamente deve molto all'intervento del Boccaccio, ma che non necessariamente deve al Certaldese la sua stessa esistenza.

In questo senso gli sviluppi critici di maggiore interesse si devono agli studi del Balduino il quale, per l'appunto, ha cercato di indagare la questione affrontandola più dal punto di vista dei cantarini che da quello del Boccaccio. Si tratta di valutare l'evoluzione di un metro interpretandola per quello che la tradizione stessa ci offre; in definitiva, muovendosi dalla considerazione, per dirla con le parole dello stesso Balduino, che «una nuova forma metrica non è, di norma, creazione ex nihilo, che come tale [... ] nasca improvisa e spontanea dalla fantasia di un singolo artista; è piuttosto un organismo che, venendo ad inserirsi in un "sistema", muove da una o più forme preesistenti e si presenta, rispetto ad esse, come innovazione parziale». ${ }^{6}$ La questione implicita resta, ancora una volta, quella relativa alla struttura metrica della tradizione canterina preboccaccesca, soprattutto dopo che rinnovati sforzi linguisticofilologici sul primo cantare in ottave non boccaccesco, il Cantare di Fiorio e Biancifiore, mostrano come esso ben difficilmente possa essere considerato come dipendente dall'opera del Boccaccio. ${ }^{7}$ In effetti le ricerche del Balduino, e parallelamente quelle del D e Robertis, sembrano indicare con buona approssimazione che per il Cantare di Fiorio e Biancifiore si debba parlare «di un'opera nata in area veneta e di lì diffusasi, in un secondo tempo, in altre zone d'Italia». ${ }^{8}$ Seppure, quindi, non possiamo esserne assolutamente sicuri, certo è che disegnare lo sviluppo della tradizione canterina lungo un percorso di diretta discendenza del Cantare di Fiorio dal Boccaccio appare quanto mai avventuroso. Lo stesso Balduino, d'altra parte, ha messo in rilievo, con una certa efficacia argomentativa, come alcune tracce petrarchesche nel Filostrato del Boccaccio possano indurci a spostare la datazione di tale opera verso il 1339 (contro l'ipotesi del Ricci appoggiata anche dal Branca, che lo datava 1335), ulte riormente stringendo, perciò, i limiti entro cui muoversi per un'eventuale ed a questo punto improbabile ipotesi di discendenza del cantare dal poema del Boccaccio. ${ }^{9}$

Eppure, se questa parte dell'argomentazione del Balduino appare in larga misura condivisibile, assai più discutibile risulta il seguito del ragionamento. Secondo il Balduino, infatti, tale indicazione potrebbe «indurci a ridimensionare e a rimettere in discussione, per un'importante e magari aurorale fase della nostra poesia cavalleresca e leggendaria, la tradizionale prospettiva florentinocentrica [... ] Al limite, per ciò che è del metro, già di qui potrebbe sorgere

6. Cfr. Armando Balduin o, Boccaccio, op. cit., p. 98.

7. Su questo punto in particolare, soprattutto per un invito a rinnovare gli sforzi di interpre tazione storica e metrica dei cantari toscani preboccaceschi, cfr. Antonio FRAN CESCH ETTI, «Rassegna di studi sui cantari», Lettere italiane, n. 25, 1973, p. 556-574.

8. C fr. Armando Balduin o, B occaccio, op. cit., p. 110, e cfr. anche D omenico D e Robertis, «C antari antichi», Studi di filologia italiana, n. 28, 1970, p. 67-175.

9. Cfr. Armando Balduin 0, Boccaccio, op. cit., p. 231-247. 
I'invito a rivedere il tradizionale itinerario da sud a nord, fino magari ad invertirne il senso di marcia». ${ }^{10} \|$ che significa cercare di recuperare certi pregiudizi della scuola storica di cui gli studi del D ionisotti, prima, e del D e Robertis, poi, avevano ormai fatto giustizia. Può darsi anche che il Balduino abbia ragione: può darsi, cioè, che i termini storico-geografici della questione del primo sviluppo della tradizione canterina vadano rivisti. Tuttavia quello che lascia perplessi è la strategia argomentativa. N onostante, infatti, la premessa metodologica di un lavoro incentrato sull'ipotesi di una forma metrica comeappartenente ad un sistema, Balduino vorrebbe che tutta l'interpretazione relativa alle origini dell'ottava rima e della tradizione canterina venisse completamente rivista sulla scorta della supposta precedenza cronologica del Cantare di Fiorio e Biancifiore rispetto al Boccaccio. Q uello che non si dovrebbe attribuire al Boccaccio, insomma, lo dovremmo invece attribuire all'anonimo del Cantare di Fiorio e Biancifi ore. D 'altra parte, i rischi di tale procedimento si avvertono già in questa considerazione del Balduino circa il Cantare di Fiorio e Biancifiore che, mentre vorrebbe correggere la posizione del Dionisotti e del De Robertis in merito ai criteri di datazione dei cantari e le possibilità interpretative lasciate al critico moderno, finisce invece, a nostro avviso, per ribadirne la coerenza interna del ragionamento: ${ }^{11}$

In discussione non è la scelta indebita fra l'una e l'altra variante, l'aggiunta o rielaborazione di questa o di quella stanza; si tratta invece dell'arduo, magari al eatorio, ma non sempre illegittimo tentativo d'individuare il momento storico in cui un testo di codesto genere cominciò a vivere come organismo in sé compiuto, e dotato di una sua fisionomia metrica, che non è detto (anzi non è dimostrato affatto) dovesse mutare radicalmente prima di giungere alle redazioni a noi note. Si provi d'altronde ad applicare le regole fissate per la datazione dei cantari a quella di opere non solo più illustri, ma anche debitamente firmate e in genere, tra l'altro, trasmesse a noi da un numero di copie incomparabilmente più alto: tanto per cominciare, ad esempio, il Cantare di Fiorio e Biancifiore precederebbe di oltre un trentennio la parte più antica della produzione canterina di Antonio Pucci, e sorte non dissimile toccherebbe al Filostrato; ma sarebbero ancora circostanza fortunate, se è vero che, mentre il Filocolo andrebbe situato a fine Trecento, Caccia di D iana e Amorosa Visione sarebbero inevitabilmente catalogate come prodotti quattrocenteschi, il N infale Fiesolano sarebbe magari registrato con la data 1414, e cosi via. Come non sospettare, allora, che anche e soprattutto l'impenetrabile anonimato che per lo più li avvolge, abbia giocato - per numerosi cantari - a sfavore di una più equa collocazione storica?

La citazione, così estesa, crediamo indichi con sufficiente chiarezza quali sono i pericoli dell'impostazione del Balduino, giacché se appare del tutto legittimo che il critico si sforzi «nel tentativo d'individuare il momento storico in

10. Ibid., p. 112.

11. Ibid., p. 108-109. 
cui un testo di codesto genere cominciò a vivere come organismo in sé compiuto, e dotato di una sua fisionomia metrica»; non altrettanto si può dire quando tale «tentativo» si elabora affidandosi ai sospetti, alle ipotesi. N é, d'altra parte, pare necessario insistere sulla seconda parte delle osservazioni del Balduino, quella cioè relativa ai criteri di datazione applicata ad altri testi letterari dello stesso periodo, se non per ricordare che fu la tradizione testuale, ovvero «'impenetrabile anonimato» di quei cantari che suggerì al D e Robertis la teoria (come ogni buona metodologia filologica raccomanderebbe) e non viceversa come invece sembrerebbe pensare il Balduino.

Le ricerche del Balduino hanno comunque avuto l'indubbio merito di riaprire il dibattito attorno all'origine dell'ottava rima e, ciò che è ancora più significativo, di iscrivere definitivamente tale dibattito in un discorso complessivo sull'evolversi della poesia trecentesca, per quanto riguarda sia le coordinate cronologiche, sia quelle geografiche. In questa prospettiva, ci pare che siano da segnalare due punti fondamentali su cui verte il discorso critico del Balduino e che appaiono, almeno in parte, condivisibili. Innanzitutto, come già si segnalava, la necessità di considerare la questione metricologica in un più ampio discorso storico-culturale: sicché il Boccaccio, piuttosto che l'inventore, andrà considerato il codificatore dell'ottava rima. In secondo luogo la ricerca di forme metriche affini che preannuncino in qualche misura l'affermarsi dell'ottava (in questo senso appaiono interessanti le considerazioni del Balduino circa il metro di alcuni laudari ed il rinvio quindi a lacopone da Todi).

Tuttavia non sarà forse vano tornare a discutere quello che è il terzo punto dell'argomentazione del Balduino, ovvero, per almeno un caso specifico, quello del Cantare di Fiorio e Biancifiore, I'ipotesi di un'attestazione assai alta di ottava rima narrativa in un testo di provenienza veneta. II cantare, infatti, che ci è tràdito dal codice miscellaneo M agliabechiano VIII. 1416, vergato certamente entro il 1349 (che è la data più bassa che si trova nel codice), ad un attento esame linguistico si rivela come si diceva di origine veneta. Particolarmente significativo appare al Balduino il fatto che nella chiusa del Cantare, il codice presenti la seguente variante (nostri i corsivi):

\section{E Fiorio ritornò di qua dal mare} ed arrivò nella dolce Toscana ecc.

Laddove tutti gli altri codici, con l'eccezione del quattrocentesco Parigino $\mathrm{N}$ at.It. 1069, anch'esso di origine toscana, tralasciano il riferimento alla Toscana e piuttosto accennano, più genericamente, ad un ritorno dell'eroe «alla sua casa». Sicché, tale annotazione parrebbe essere un adattamento del tema del Cantare al nuovo pubblico toscano e quindi, presumibilmente, ci costringerebbe a retrodatare la composizione del Cantare di almeno qualche anno, ovve ro il tempo necessario affinché un'opera scritta in Veneto raggiungesse il successo e fosse quindi diffusa, riadattata, anche in area toscana. Eppure, proprio questo caso pare sintomatico delle difficoltà precisamente metodologiche nelle 
quali incorre il ragionamento del Balduino. II riadattamento toscano, infatti, potrebbe aver portato con sé una modifica della forma metrica originaria: sicché l'attestazione dell'ottava rima, rimane, fino a prova contraria, comunque un'attestazione toscana e non veneta. Tanto più che casi analoghi non mancano. Ad esempio, nella cosiddetta Spagna «maggiore», largamente ispirato alla narrazione dell'Entreée d'E spagne, e legata alla formulazione che di tali vicende si ha nella Prise de Pampaleune, leggiamo nel canto XXV, lassa 46 (nostri i corsivi):12
Disse il re D esider: - I' vo' da Carlo
mezo il tesoro che ci s'è rubato,
sicché io possa alla mia gente darlo;
e Toscani e Lombardi in ogni lato
il brando a lor voler possin portarlo, voglin al collo o voglin cinto al lato; e voglio che ' $n$ Toscana e L ombardia doppo mia morte già mai più re non sia - .

O ra, come ha avuto modo di osservare Corrado Bologna, «la zeppa evidente dei "Toscani", accanto ai "Lombardi" della Prise sembra assumere i tratti di un'intenzionale riplasmazione ideologica». ${ }^{13} \mathrm{~N}$ é può sfuggire che tale riplasmazione (perpetrata, si badi, attraverso la stessa strategia di riscrittura che si è vista in atto nel Cantare di Fiorio e Biancifiore) porta con sé un adattamento metricologico, ovvero l'introduzione dell'ottava rima. Per cui, data la situazione testuale e a meno di non voler fare anche della Spagna «maggiore» - e di tutti gli altri casi analoghi - una testimonianza indiretta della presenza di ottave rime in area veneta, l'ipotesi del Balduino rimane appunto solo un'ipotesi: tanto affascinante, quanto metodologicamente discutibile. N el contesto di una tradizione, quale la canterina, che si profila estremamente caratterizzante e che si svolge e definisce nelle oscillazioni fra oralità e scrittura, i dati della paleografia, giusta la lezione del D e Robertis, rimangono gli unici ad offrire sufficienti garanzie argomentative, giacché ogni riscrittura comporta appunto un riaggi ustamento del testo, dal quale neppure la fisionomia metrica esula. Sicché un testo scritto in ottave in area toscana alla metà del Trecento, ci potrà informare circa la situazione della letteratura canterina in quell'ambito geografico ed a quell'altezza cronologica, ma non presuppone affatto un antigrafo dalla stessa fisionomia, né per ciò che riguarda le lezioni del testo, né per la sua veste metrica.

La questione dell'origine dell'ottava rima, in definitiva, non ruota tanto attorno alla retrodatazione dei cantari in ottave ad una data più alta della com-

12. C fr. M ichele Catalano (a cura di). La Spagna. Poema cavalleresco del secolo XIV, 3 voll., Bologna: Commissione per i testi di lingua, 1939-40, II , p. 372.

13. Cfr. Corrado Bo Lo Gn A, «La letteratura dell'Italia settentrionale nel Trecento», in Letteratura italiana, Storia e geogfrafia. I. L'età medievale, Torino: Einaudi, 1987, p. 511-600, p. 553. 
posizione dei poemi boccacceschi; piuttosto, essa deve rivedersi nella valutazione del Filostrato e del Filocolo, in primis, nell'ambito dell'evolversi di un metro - l'ottava rima, appunto - che si affermò, sulla pagina, solamente dopo l'esperienza poetica del Boccaccio. Certamente, questo non comporta che il C ertaldese ne fu senz'altro l'inventore: etuttavia significa che nell'ambito di una tradizione che con ogni probabilità si sviluppava, su di una «matrice oitanica, attraverso i canali della diffusione colta e di quella canterino-giullaresca», 14 il Boccaccio andrà considerato il grande mediatore equindi, se non altro pro forma, proprio a lui spetterà il titolo di pater dell'ottava rima. 